\title{
Una mirada al abordaje de la corrupción en Argentina
}

\author{
Germán E. Oestmann \\ germaneoestmann@gmail.com \\ Universidad Nacional del Chaco Austral \\ Presidencia Roque Sáenz Peña, Chaco - Argentina
}

\section{RESUMEN}

El objetivo del presente trabajo fue realizar un análisis del fenómeno de la corrupción y sus consecuencias en las sociedades democráticas. Para lo cual se realizó una búsqueda de información sobre sus generalidades y consecuencias. La corrupción afecta negativamente los fines públicos perseguidos en un Estado Constitucional Social de Derecho, perturbando económicamente el patrimonio de una nación, así como el bienestar de la sociedad, lo cual incide negativamente en la calidad de vida de sus ciudadanos, así como en sus derechos humanos, sociales y económicos.

Palabras clave: corrupción; dimensiones y consecuencias; Argentina. 


\title{
A look at the approach to corruption in Argentina
}

\begin{abstract}
The objective of this work was to analyze the phenomenon of corruption and its consequences in democratic societies. Therefore, a search for information was carried out on its generalities and consequences. Corruption negatively affects the public purposes of the Social Constitutional State of Law, economically disturbing the heritage of a nation, as well as the well-being of society, which negatively affects the quality of life of its citizens, as well as their human rights, social and economic
\end{abstract}

Keywords: corruption; dimensions and consequences; Argentina.

Artículo recibido:.10 Agosto. 2021 Aceptado para publicación: 07. Setiembre. 2021 Correspondencia: germaneoestmann@gmail.com Conflictos de Interés: Ninguna que declarar 


\section{INTRODUCCIÓN}

En 2004, la Organización de las Naciones Unidas señaló la gravedad de los problemas y las amenazas que plantea la corrupción para la estabilidad y seguridad de las sociedades al socavar las instituciones y los valores de la democracia, la ética y la justicia. Aunque el término corrupción es oído frecuentemente, no siempre se presenta una definición clara para su entendimiento, ya que su comprensión está relacionada tanto con el aspecto moral, de los valores que establece la sociedad, como con el jurídico, especialmente con costumbres sociales, robo, hurto, enriquecimiento ilícito, favorecimiento indebido, soborno, propina, etc. (Mileski, 2011; Martínez Cárdenas y Ramírez Mora, 2020; Katz y col, 2017). Diversos estudios indican que la corrupción, así como la violencia y la avaricia, es una condición de la naturaleza humana influenciada por el medio ambiente: familia, religión, educación, cultura, formación, etc., generando cambios en la condición humana, que en general tienden a un beneficio propio (Batista, 2005). Seña (2002), señala que son actos corruptos aquéllos, llevados a cabo por dos partes, que implican la violación o transgresión de forma activa o pasiva de un deber posicional o de la función que cumple. El autor señala que esto se produce bajo un sistema normativo que le sirva de referencia, y están vinculados a un beneficio extra posicional, cuyos actos se realizan generalmente bajo discreción o desconocimiento de la sociedad.

En este sentido y según el Índice de Percepción de la Corrupción 2018 (IPC) elaborado por la Organización Transparencia Internacional (TI), la Argentina mejoró en los últimos años su ubicación lo que puede atribuirse a la "Leyes anticorrupción vigentes". Si bien en el IPC 2019, el país volvió a incrementar su puntuación, el 49\% de los ciudadanos señaló su posición sobre un aumento de la corrupción en el país en los últimos 12 meses; el 13\% señaló que experimentaron extorsión sexual o conocen a alguien que pasó por esta situación; el 19\% pagó algún soborno por servicios; el 21\% indicó que se le ofrecieron sobornos a cambio de votos (Coralie Pring, 2019). En el IPC 2020, Argentina empeoró su puntuación, atribuyéndose ello -al igual que en la mayoría de los países- a la percepción social respecto de la gestión de la crisis sanitaria expuesta por la pandemia Covid-19 (Transparencia internacional, 2020). Por lo tanto, en el presente artículo realizaremos un análisis del fenómeno de la corrupción y sus consecuencias en las sociedades democráticas.

\section{ESTRATEGIAS METODOLÓGICAS O MATERIALES Y MÉTODOS}


La información se recopiló accediendo a distintas fuentes de información provenientes de bases de datos como Scielo, Google Académico y Redalyc; también legislación, tesis y revistas digitales. Las palabras empleadas como descriptores para la búsqueda fueron: corrupción, sector público, causas y consecuencias de la corrupción. La información se compiló sin límite de tiempo, o cobertura geográfica, seleccionándose los documentos relacionados a la temática. La bibliografía se categorizó a fin de abarcar el tema desde sus generalidades a sus consecuencias.

\section{RESULTADOS Y DISCUSIÓN}

\section{Generalidades de la corrupción}

La noción de corrupción está generalmente asociada a la violación del deber de probidad del funcionario en su enriquecimiento personal, el cual no sólo comprende el enriquecimiento económico. Puede definírsela como la desviación de la conducta de un funcionario público, que se aparta de las normas establecidas para ponerse al servicio de intereses privados (Huntingto, 1972). La forma en cómo se reparte y ejerce el poder público es una función entendida de la administración pública, debiendo ser dinámica y objetiva de manera que se distribuya en forma equitativa y correcta entre los ciudadanos, atendiendo a sus derechos fundamentales.

Sin embargo, los actores de la corrupción involucran el intercambio entre dos voluntades, la transgresión de una relación de confianza o la existencia de un cierto poder de discrecionalidad de parte de quien ejecuta el comportamiento corrupto, en general son internos, propios del sistema o asimilados por el mismo y sus funcionarios sin importar el cargo (Ferreira, Ruíz Díaz y Kunert, 2019). Para Orellana, (2003) se pueden presentar seis formas distintas de corrupción: la micro corrupción, que es aquella que realizan los funcionarios (por ejemplo el robo de material de oficina); la que realizan los jefes (por ejemplo en la apropiación de los fondos públicos cuya administración les ha sido confiada); la que ejecuta una asociación delictiva en la que intervienen jefes y funcionarios; la realizada por una asociación entre usuario y funcionario; la ejecutada por una asociación entre usuario y jefe; y, por último, la que es llevada a cabo por una asociación entre usuarios, funcionarios y jefes.

La tipología de las prácticas corruptas es resumida por Gilli (2014), quien menciona el soborno, el tráfico de influencias y cooptación del Estado, como algunas de ellas. Además, el autor citando a Sautu, (2004), proporciona una clasificación más amplia de 
los actos corruptos mencionando transferencias patrimoniales, otorgamiento de privilegios, abuso de poder, favoritismo y clientelismo, sobornos, extorsión, arreglos, alteraciones fraudulentas del mercado, uso de e información privilegiada, entre otros. El conocimiento de las prácticas corruptas es una condición necesaria pero no suficiente para el combate de la corrupción sistémica, siendo necesario reconocer las tramas delictuales e identificar sus debilidades a los efectos de operar sobre las mismas, con muy pocas condenas al respecto (Labarqui, 2003; Piana y Arévalo, 2019).

\section{Factores que inciden en la corrupción}

Entre los factores que inciden en la corrupción y que la teoría señala como relevantes se destacan la cultura, el nivel de desarrollo, el grado de conflictividad social y las instituciones políticas y económicas, los que fueron abordados por Labarqui (2003). El autor buscó verificar el poder explicativo de estos factores, y en particular, analizar la relación entre el tipo de régimen y el nivel de corrupción, así como también la relación entre libertad económica y corrupción. Encontró que el nivel de desarrollo condiciona fuertemente la calidad de la burocracia estatal tanto en lo que hace a las formas de reclutamiento, su profesionalidad, su estabilidad y su nivel de remuneración. Además, señala que la democratización no necesariamente implica una reducción automática de la corrupción, ya que en un principio la democracia puede estar asociada a una mayor corrupción, ya sea que ésta sea una apariencia producto de la mayor libertad de prensa existente en las democracias, ya sea que se trate de un aumento real fruto de actividades inherentes a la democracia que pueden dar lugar a actos de corrupción (como por ejemplo las campañas electorales). Sin embargo, destaca que países que integran herramientas como la transparencia, rendición de cuentas, participación ciudadana y libertad económica en su gestión efectivamente llevarían a una caída en los niveles de corrupción, disminuyendo oportunidades de corrupción. Señala, además, que diferencias geográficoculturales son relevantes en materia de corrupción, ya que países que han transitado recientemente del autoritarismo a la democracia y que poseen economías altamente reguladas al mercado presentan mayores niveles de corrupción.

Miranzo Díaz (2018), señala entre las causas subjetivas de corrupción la vanidad, el egoísmo, la satisfacción personal, el ansia de poder o incluso el reconocimiento social. Estas conductas llevan la pérdida de confianza en la función pública, ya que quien ejerce el acto corrupto carece de valores éticos, honestidad, responsabilidad, o el sentido de 
interés público y de la Administración como salvaguarda de los derechos e intereses de los ciudadanos, y que pueden explicarse también desde la psicología (Julián y Bonavia, 2020). Además, la corrupción es una conducta de riesgo (Ackerman y Palifka, 2016), ya que quien la ejerce debe esquivar obstáculos como restricciones legales, controles internos y externos, la posibilidad a ser delatado por compañeros u otras personas parte del proceso, etc., pudiendo o no tener el resultado esperado del acto llevado a cabo (Fernández Ríos, 1999). En relación a esta elevada tasa de reiteración que se observa en los trabajadores que alguna vez cometen este tipo de irregularidades, es precisamente un sentimiento fortalecido de impunidad, de estar por encima de las leyes, las instituciones, y el resto de elementos de control del estado, como a falta de control efectivo y trabas previas a la corrupción (Miranzo Díaz, 2018). Un ejemplo de esto puede ser la extorsión, mediante la que se les exige pagos extras a los contribuyentes, pagos extras para brindar servicios públicos, como, por ejemplo, la emisión de pasaportes, y desde los jefes de los funcionarios que les cobran "rentas" a sus propios subordinados requiriéndoles una cierta cantidad fija por mes o semana. Otro ejemplo es el abuso del poder discrecional burocrático, entre las cuales son llevadas a cabo por ministros que "venden" su poder; oficiales que embolsan porcentajes de los contratos gubernamentales, transfiriendo el dinero a cuentas bancarias en el exterior; funcionarios que consiguen contratos de gobierno para sí mismos, y partidos políticos que utilizan la perspectiva de llegar al poder y obtener "rentas" de negocios internacionales a cambio de contratos gubernamentales (Wainstein, 2003).

La fortaleza del marco normativo en el que actúa la corrupción es un factor esencial para conseguir la erradicación de la misma de la Administración Pública en general y de los contratos públicos en particular, por lo que un sistema normativo débilmente construido posibilita, e incluso favorece, los comportamientos irregulares (Simancas Simancas, 2010). Además, para que el derecho penal cumpla con sus funciones preventivas debe tener la suficiente capacidad motivante como para disuadir a quienes están dispuestos a afectar los bienes que el legislador considera dignos de ser protegidos (Seña, 2016). La debilidad de los procedimientos y mecanismos institucionales, con procedimientos débiles e incompletos, sin bases en los principios de publicidad y transparencia, hacen que, aunque un Estado cuente con exhaustivas leyes administrativas y penales para 
sancionar y prevenir la corrupción, estas no sean respetadas, haciendo vulnerables los mecanismos institucionales eficientes de control, monitoreo y buena gobernanza.

La baja profesionalización del sector público, es decir, de los encargados de diseñar, dirigir y participar en un contrato público u otros procedimientos administrativos es una de las mayores puertas hacia la corrupción. Esto se produce principalmente por carencia especifica de los políticos sobre conocimientos específicos de la gestión pública que se requieren en procesos administrativos complejos como la contratación pública, y adicionalmente tienden a padecer una mayor exposición a los conflictos de intereses, viéndose afectados con mayor facilidad por intereses partidistas o particulares (Kiltgaard, 2009). También, la falta de medios técnicos o humanos, en las pequeñas entidades públicas hace que los funcionarios o empleados públicos en cuestión no tengan capacidad para especializarse en una materia, al tiempo que se reduce el número de personas encargadas de gestionar los procedimientos. Esto facilita la participación de las clases políticas de manera directa, y por otro, la práctica de sobornos, favores, etc., debido a la facilidad para detectar el centro de decisión en el desarrollo de un procedimiento, ya que basta con sobornar una o dos personas en las que el poder decisorio se encuentra centralizado. De modo que la existencia de un cuerpo administrativo de profesionales públicos, con carácter de permanencia, constituye uno de los mejores medios para la prevención de la corrupción (Villoria Mendieta y Izquierdo Sánchez, 2016).

La falta de independencia de los gestores y organismos de control, dada por una excesiva dependencia de sus órganos superiores, especialmente de los altos cargos políticos, impiden el correcto funcionamiento de los mismos, ocasionando una gran inseguridad jurídica e inestabilidad en los gestores públicos. Esto puede condicionar gravemente los controles realizados por figuras internas a la Administración, encargadas de evaluar con carácter previo determinadas actividades de la entidad. Además, la falta de medios técnicos y humanos, pueden dificultar las tareas de intervención y auditoría, así como las obligaciones de transparencia y publicidad. También, la falta de mecanismos legales que doten de claridad y de herramientas a los diferentes actores dentro del proceso de contratación, y que faciliten una actuación íntegra de los mismos y un control efectivo, y no únicamente formal de las actuaciones de las entidades públicas, suponen un grave contratiempo en la lucha contra la corrupción (Seña, 2016). 
La descentralización del gasto público, hacia la privatización y descentralización de la gestión política, democrática y económica, afecta a la gestión de fondos públicos, de manera que el poder decisorio de la función pública se encuentra, en multitud de entidades adjudicadoras de diferente tamaño, que son las encargadas de adjudicar los contratos y distribuir el gasto público, asimilándose más a un modelo empresarial que a la tradicional línea jerárquica de la Administración (Bautista, 2007). Esto crea nuevos incentivos e intereses generales localistas, originando una situación de contrato de agencia en la que el agente (el poder adjudicador), puede diferir en sus intereses de aquellos del principal (el Estado, otras Administraciones Públicas, etc.) y que pueden dar lugar a conflictos de intereses contrapuestos. También genera la descentralización de la Administración Pública una "pérdida de control" de la actividad de los poderes adjudicadores -debido a la dificultad que para el control de la actividad pública supone la multiplicación de entidades a las que fiscalizar, afectando el poder y la capacidad para controlar y monitorear las decisiones del agente (Bardham y Mookherjee, 2006).

\section{Las consecuencias de la corrupción}

La corrupción es un fenómeno que se reproduce ante la precariedad y fragilidad de lo público y del sentido de pertenencia hacia ello, que no se percibe únicamente en la administración de los recursos públicos, sino también en todos los procesos democráticos y sociales (Arteaga, 2005). Rodríguez Arana (1996) afirma que posiblemente el efecto más perverso de la corrupción sea el falseamiento del mismo sistema democrático y la consolidación de una forma de vida más bien alejada de los hábitos y virtudes propias de la democracia, ya que afecta principios de objetividad, legalidad, transparencia y rendición de cuentas (Diez Getino y Torija Herrero, 2016).

Las prácticas corruptas crean un canal alternativo para la tramitación de los diferentes procedimientos y expedientes administrativos, que, mediante el abuso de fondos públicos, la adquisición de privilegios indebidos y la generación de nuevas reglas del juego, sustituyen y destruyen el entramado procesal jurídico-democrático, atentando directamente contra el Estado de Derecho y la eficiencia gubernamental (Villoria Mendienta, 2006; Bautista, 2007). Afecta también afecta al propio proceso de creación y planificación de las políticas públicas y puede culminar en un efecto sobre el comportamiento electoral de los votantes, ya que la lleva a la desafección y pérdida de confianza en las instituciones, en el Gobierno, y en el Sector Público en general (Lizcano 
Álvarez y Villoria Mendieta, 2013). El surgimiento de ideales políticos y con ellos la denominada política de confianza, a favor de la credibilidad, veracidad, y honestidad de los políticos, independientemente de la ideología que profesen, hace que aumente la confianza de la población en la Administración, como así en sus representantes políticos, las instituciones y la gestión pública (Thompson, 2001).

Por otra parte, el Banco Mundial ha afirmado que la corrupción es uno de los mayores obstáculos para el desarrollo económico y social, dado que debilita el Estado de derecho y las bases institucionales de las que depende el crecimiento (Mccarthy, 2015). La corrupción tiene efectos devastadores en el mercado y la eficiencia del mismo (Salinas Jiménez y Salinas Jiménez, 2001). En este sentido, la corrupción puede incidir directamente en el ciudadano a través del aumento de los precios en los bienes y servicios producidos por las empresas inmersas en corrupción (Diez Getino y Torija Herrero, 2016). También afecta la toma de decisiones en la estrategia de gasto público. De esta forma, se contratan servicios e infraestructuras innecesarias, guiadas por intereses corruptos - generalmente grandes infraestructuras de difícil evaluación y control (Mauro, 1998). Se produce, en definitiva, una pérdida de oportunidad para la sociedad y para los intereses generales, ya que dichos fondos, invertidos en aspectos innecesarios, dejaron de utilizarse para cubrir otras necesidades esenciales del Estado del Bienestar (Holmes, 2015).

\section{CONCLUSIÓN O CONSIDERACIONES FINALES}

La corrupción, es un fenómeno que tiene innegables consecuencias negativas, tanto desde una perspectiva utilitarista, como ética ya que supone una traición al deber, a la honradez y a la integridad en las funciones públicas. Socaba los cimientos de la misma civilización, del Estado de Derecho y del sistema democrático y económico actual. La búsqueda de métodos eficaces para combatir la corrupción, es esencial para la promoción del desarrollo económico, calidad de la democracia y de la justicia social. Estructuralmente los efectos de la corrupción son acentuados en el desarrollo económico del país, más aún para un desarrollo económico sostenible, ya que afecta también a las instituciones incentivadoras.

La corrupción representa un obstáculo a la concretización de los fines públicos perseguidos en un Estado Constitucional Social de Derecho, afectando económicamente el patrimonio estatal y socialmente el interés general, por su impacto negativo y 
destructivo sobre la calidad de vida de la población y los derechos humanos, sociales y económicos.

\section{LISTA DE REFERENCIAS}

Ackerman, R.S. y Palifka, B.J. (2016). Corruption and Government: Causes, Consequences, and Reform. Cambridge: Cambridge University Press. https://doi.org/10.1111/gove.12273

Alejandro Katz ... [y col.]; compilado por Secchi, P., Kalpschtrej, K.; Arcidiácono, P. (2017). Poder Ciudadano: corrupción y transparencia: informe 2016-2017/. 1a ed. Ciudad Autónoma de Buenos Aires: Eudeba. Recuperado de: https://poderciudadano.org/wpcontent/uploads/2020/12/InformePoderCiudadano2017.pdf

Arteaga A.M. (2005). ¿Es la corrupción la causa de la pobreza? En: Corrupción y derechos humanos: estrategias comunes por la transparencia y contra la impunidad. IPC, Instituto Popular de Capacitación: Medellín.

Bautista, O.D. (2007). La ética en la gestión pública: fundamentos, estado de la cuestión y proceso para la implementación de un sistema ético integral en los gobiernos. Universidad Complutense de Madrid. Madrid, pp. 215-225. Recuperado de: https://eprints.ucm.es/id/eprint/7529/

Bardham, P. y Mookherjee, D. (2006). Decentralization, corruption and government accountability. En ROSE-ACKERMAN, S (edit.). International Handbook on the Economics of Corruption. Northampton (EEUU): Eduard Elgard Publishing.

Coralie Pring, J.V. (2019). Transparencia Internacional. Barómetro Global De La Corrupción En América Latina y El Caribe 2019. Aumento de la corrupción por país. Porcentaje de personas que sostienen que la corrupción aumentó en los últimos meses. 12 Recuperado de: https://www.transparency.org/files/content/pages/2019_GCB_LAC_Full_Report ES.pdf

Diez Getino, J.E. y Torija Herrero, S. (2016). Modelo de estrategia para fomentar la integridad y prevenir la corrupción. Revista Internacional transparencia e integridad, $\quad$ R.I.T.I., 2 Recuperado de: http://revistainternacionaltransparencia.org/wpcontent/uploads/2016/12/Jos\%C3\%A9-Enrique-Diez-y-Susana-Torija.pdf 
Fernández Ríos, L. (1999). Psicología de la Corrupción y los Corruptos. Santiago de Compostela: Grupo Editorial Universitario.

Ferreira, S., Ruíz Díaz, E., y González Kunert, L. (2019). Corrupción: Revisión, dimensión social de la corrupción y esfuerzos en materia legal en el Paraguay. Ciencia Latina Revista Científica Multidisciplinar, 3(1), 186 - 214. Recuperado a partir de https://ciencialatina.org/index.php/cienciala/article/view/18

Gilli, J.J. (2014). La corrupción: análisis de un concepto complejo. Revista de Instituciones, Ideas y Mercados, 61: 39-63. Recuperado de: https://www.eseade.edu.ar/images/RIIM_60/gilli_riim61.pdf

Huntingto, S.P. (1972). El orden político en las sociedades en cambio. Paidós, Buenos Aires.

Holmes, L. (2015). Corruption: A Very Short Introduction. Oxford: Oxford University Press.

Julián, M, \& Bonavia, T. (2020). Variables psicológicas asociadas a la corrupción: una revisión sistemática. Anales de Psicología, 36(2), 330-339. Epub 14 de diciembre de 2020. https://dx.doi.org/10.6018/analesps.36.2.389371

Kiltgaard, R. (2009). Corrupción Normal y Corrupción Sistémica. Banco Interamericano de Desarrollo. Recuperado de: https://publications.iadb.org/handle/11319/1345

Labaqui, I. (2003). Las Causas de corrupción: un estudio comparado. Colección, 14, 155196.

Lizcano Álvarez, J. y Villoria Mendieta, M. (2013). Corrupción y transparencia: Aspectos contextuales, metodológicos y empíricos. Revista de Responsabilidad Social de la Empresa, 14: 13-48, OCDE, Competition and Procurement: Key Findings. Paris: OECD $2011 . \quad$ Disponible en: http://www.oecd.org/regreform/liberalisationandcompetitioninterventioninregula tedsectors/48315205.pdf

Martínez C., E.E. y Ramírez Mora, J.M. (2010). La corrupción en la Administración Pública: un perverso legado colonial con doscientos años de vida republicana. Reflexión

Política,

12(23):

68-80.

https://www.redalyc.org/articulo.oa?id=11015102006 
Mccarthy, L. (2015). Un movimiento mundial contra la corrupción ¡está ocurriendo ahora. En la página web del Banco Mundial. Disponible en:

http://blogs.worldbank.org/voices/es/un-movimiento-mundial-contra-la-corrupcionesta-ocurriendo-ahora

Mileski, H.S. (2011). Tesis doctoral sobre las Transformaciones del Estado y las nuevas exigencias del control de la corrupción: transparencia fiscal, control administrativo y control social. Tesis Doctoral. Facultad de Derecho. Departamento de Derecho Administrativo, Financiero y Procesal. Universidad de Salamanca. España. Recuperado de: https://gredos.usal.es/handle/10366/110542

Miranzo Díaz, J. (2018). Causas y efectos de la corrupción en las sociedades democráticas. Revista de la Escuela Jacobea de Posgrado, 14: 1-26. Recuperado de: https://www.jacobea.edu.mx/revista/numeros/numero14/1.Javier-MiranzoDi\%CC\%81az-Causas-Efectos-Corrupcion-Sociedades-Democraticas.pdf

Naciones Unidas. Oficina De Las Naciones Unidas Contra la Droga y el Delito. Convención De Las Naciones Unidas Contra La Corrupción. Naciones Unidas, Nueva York, 2004. Recuperado de: https://www.unodc.org/documents/mexicoandcentralamerica/publications/Corru pcion/Convencion_de_las_NU_contra_la_Corrupcion.pdf

Orellana, P., (2003). Corrupción y probidad: problemas y soluciones, en Comunidad Virtual de Gobernabilidad, Desarrollo Humano e Institucional. Santiago, Chile.

Piana R. S., \& Arévalo M. (2019). Precedentes jurisprudenciales en la evaluación de la corrupción en la Argentina. Un estudio a partir de tres casos judiciales. Estudios De Derecho, 77(169), 13-34. DOI: 10.17533/udea.esde.v77n169a01

Rodríguez Arana, J. (1996). Ética Institucional: Mercado "Versus" Función Pública. Madrid: Dykinson y Escuela Gallega de Administración Pública.

SALINAS JIMÉNEZ, J. y SALINAS JIMÉNEZ, M. M. (2001). Efectos de la corrupción sobre la productividad: un estudio empírico para los países de la OCDE”. Instituto de Estudios Fiscales y Universidad de Extremadura. P. T. N.o 6/06:2-44. Recuperado de: https://www.ief.es/docs/destacados/publicaciones/papeles_trabajo/2006_06.pdf 
Sautu, R. (2004). Catálogo de prácticas corruptas, Buenos Aires. Lumière y Laporta, Francisco J. y Álvarez, Silvina (eds.) (1997), La corrupción política, Madrid: Alianza Editorial.

Seña, J.F.M. (2002). La corrupción. Aspectos éticos, económicos, políticos y jurídicos. Barcelona: Editorial Gedisa, S/A.

Seña, J.F.M. (2016). La corrupción. Algunas consideraciones conceptuales y contextuales. Revista Vasca de Administración Pública, 104(2): 25-41. Recuperado de: https://apps.euskadi.eus/t59auUdaWar/R2/verArticulo?numejem=104\&tipo=R\& $\underline{\text { seccion }=58 \& \text { correlativo }=1 \& \text { contenido }=2 \& \text { locale }=\mathrm{es}}$

Simancas Simancas, R. (2010). El precio de la corrupción. En Temas para el Debate, 183: $19-22$.

Thompson, D.F. (2001). El escándalo político. Barcelona: Paidós.

Transparencia Internacional. (2020). Índice de percepción de la corrupción 2020 Recuperado de: https://images.transparencycdn.org/images/CPI2020_Report_ES_0802-WEB.pdf

Villoria Mendienta, M. (2006). La corrupción política. Madrid: Editorial Síntesis.

Villoria Mendieta, M. e Izquierdo Sánchez, A. (2016). Ética Pública y Buen Gobierno: regenerando la democracia y luchando contra la corrupción desde el servicio público. Madrid: Tecnos.

Wainstein, M. (2003). La Corrupción un tema para investigar. Contabilidad y Auditoría, 17. Año 9 - $\quad-\quad$ junio. Recuperado de: http://bibliotecadigital.econ.uba.ar/download/cya/cya_v9_n17_01.pdf 